\title{
A Twelve-Node Hybrid Stress Brick Element for Beam/Column Analysis
}

\author{
K.Y.Sze \\ Department of Mechanical Engineering, The University of Hong Kong \\ Pokfulam Road, Hong Kong SAR, P.R.CHINA \\ Email : kysze@hkucc.hku.hk \\ S.H.Lo \\ Department of Civil Engineering, The University of Hong Kong \\ Pokfulam Road, Hong Kong SAR, P.R.CHINA \\ Email : hreclsh@hkucc.hku.hk
}

\begin{abstract}
In this paper, a hybrid stress twelve-node brick element is presented. Its assumed stress field is derived by first examining the deformation modes of a geometrically regular element and then generalizing to other element configurations using tensorial transformation. The total number of stress modes is thirty which is minimal for securing the element rank. To reduce the computational cost associated with the fully populated flexibility matrix, the admissible matrix formulation is employed to induce high sparsity in the matrix. Popular beam bending benchmark problems are examined. The proposed elements deliver encouraging accuracy.
\end{abstract}

keywords : finite element, hybrid stress, beam bending, tall building 


\section{INTRODUCTION}

Strictly speaking, all structural members are three-dimensional. Various beam, plate and shell models were proposed to simplify the otherwise intractable three-dimensional analysis. Finite element method as a numerical tool should not be bothered by the mathematical intractability. Nevertheless, it is nearly a "must" that beam, plate and shell structures are modelled by the pertinent finite element models. Solid elements are declined because most of them suffer from locking. Recently, a number of lock-free solid elements for thin plate/shell analysis have been reported (Ausserer \& Lee 1988; Kim \& Lee 1988; Sze \& Ghali 1992, 1993; Sze, Yi \& Tay 1997). Despite of the higher computing cost as compared to plate/shell elements, these elements offer several advantages. Firstly, the problem of matching the rotational d.o.f.s in connecting different plate/shell together is avoided. Secondly, structural geometry is represented in a direct and exact manner. Hence, the surplus joint stiffness at junctions of different plate/shells can be resolved, see Fig.1. Thirdly, it is noted that a major constraint which limits the load step size in large displacement/rotation problems is the non-vectorial nature of finite rotations. This constraint no more exits when rotations are not used as kinematic variables (Park, Cho \& Lee 1995).

Fig.2 shows a typical junction of beams and columns in tall buildings. Shear walls and floor slabs are omitted for clarity. While robust eighteen-node solid finite element models for plate/shell analysis (Ausserer \& Lee 1988; Sze, Yi \& Tay 1997) can be used to model shear walls and floor slabs, twelve-node solid elements are required for the beams and columns. Our literature survey indicates that none of the advanced finite element methods including hybrid/mixed method (Pian 1964; Pian \& Sumihara 1984; Pian 1985), reduced and selective integration (Kavanagh \& Key 1972; Malkus \& Hughes 1978), incompatible displacement (Taylor, Beresford \& Wilson 1976; Simo \& Rifai 1990), assumed strain method (Bathe \& Dvorkin 1985; Huang \& Hinton 1986), stabilization method (Belytschko, Tsay \& Liu 1981; Belytschko, Wong \& Stolarski 1989) has been applied to the twelve-node element configuration. It is the purpose of the present paper to devise such an element that can deliver high accuracy in beam bending analysis.

A noticeable problem in formulating solid elements for plate/shell analysis is on ensuring a zero normal stress prediction in the transverse direction. Under pure bending and with only two nodal surfaces in the transverse direction, the thickness reduction and thickness increment on two sides of the mid-surface due to Poisson's ratio coupling will cancel each other. A plane strain state will be predicted instead of the factual plane stress state. To resolve this problem, there are at least two methods. One may artificially decouple the in-plane and out-of-plane responses in the constitutive relation with the plane stress assumption (Ausserer \& Lee 1988; Kim \& Lee 1988; Sze, Yi \& Tay 1997). Thus, the zero transverse normal strain cannot bring forward the plane strain state. Alternatively, one can employ the hybrid stress formulation (Pian 1985; Sze \& Ghali 1992,1993). 
As the twelve-node element may interface with other plate/shell elements longitudinally, the plane stress state cannot be assumed as a priori. Hence, the first method is not acceptable. The hybrid stress formulation will be adopted here.

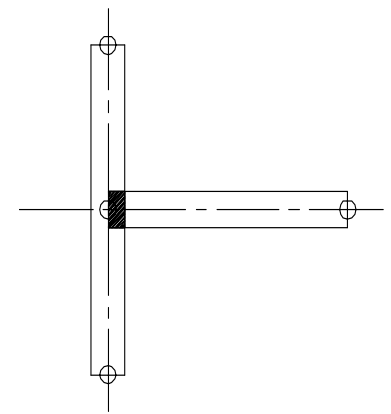

Fig.1. Joint stiffness increases as the highlighted material is accounted for in both beam or plate/shell elements

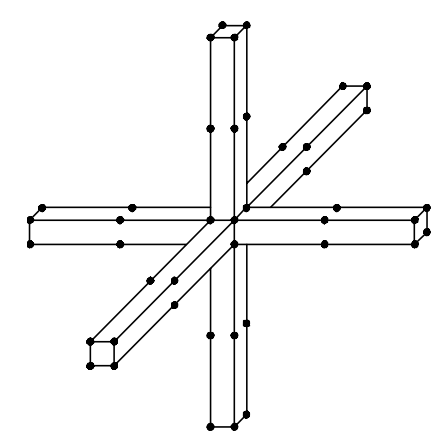

Fig.2. Twelve-node brick elements used in framework analysis

\section{GEOMETRY AND KINEMATIC OF THE 12-NODE BRICK ELEMENT}

The element under consideration is portrayed in Fig.3. The nodal interpolation functions are standard and can be written as :

$$
\begin{aligned}
& N_{1}=\xi^{-} \eta^{-} \zeta^{+}, N_{2}=\xi^{+} \eta^{-} \zeta^{+}, N_{3}=\xi^{+} \eta^{+} \zeta^{+}, N_{4}=\xi^{-} \eta^{+} \zeta^{+}, N_{5}=\xi^{-} \eta^{-} \zeta^{m}, N_{6}=\xi^{+} \eta^{-} \zeta^{m}, \\
& N_{7}=\xi^{+} \eta^{+} \zeta^{m}, N_{8}=\xi^{-} \eta^{+} \zeta^{m}, N_{9}=\xi^{-} \eta^{-} \zeta^{-}, N_{10}=\xi^{+} \eta^{-} \zeta^{-}, N_{11}=\xi^{+} \eta^{+} \zeta^{-}, N_{12}=\xi^{-} \eta^{+} \zeta^{-}
\end{aligned}
$$

where

$$
\begin{aligned}
& \xi^{-}=(1-\xi) / 2, \xi^{+}=(1+\xi) / 2, \eta^{-}=(1-\eta) / 2, \eta^{+}=(1+\eta) / 2, \\
& \zeta^{+}=\zeta(\zeta+1) / 2, \zeta^{-}=\zeta(\zeta-1) / 2, \zeta^{m}=1-\zeta^{2}
\end{aligned}
$$

$\xi, \eta$ and $\zeta$ are the parametric coordinates which vary from -1 to +1
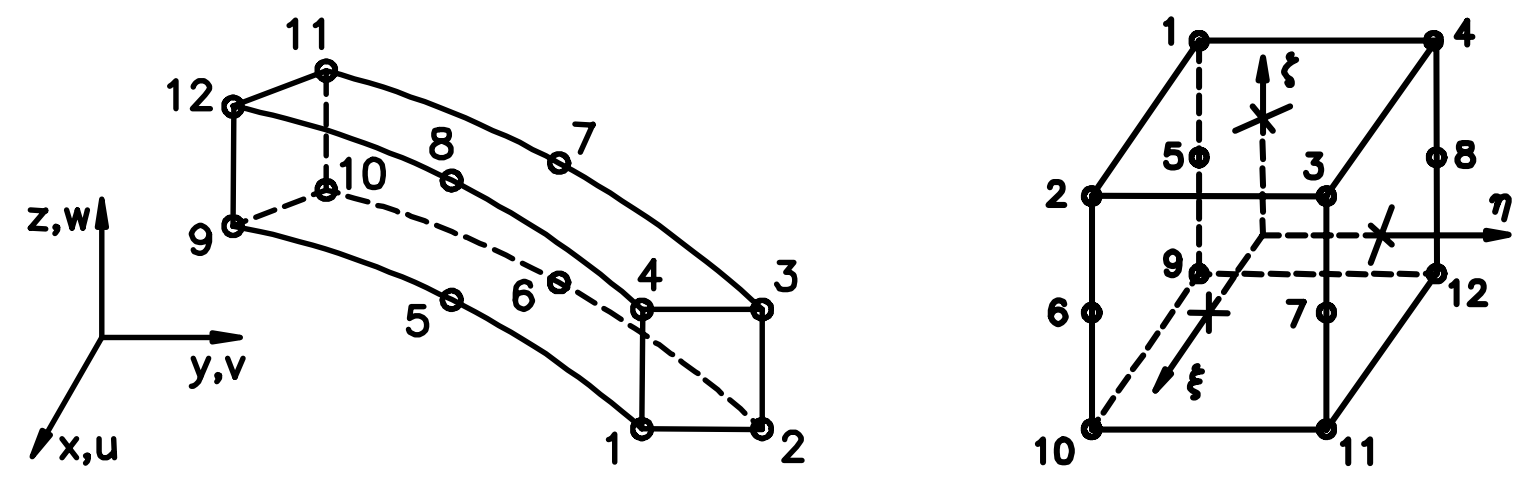

Fig.3. The 12-node element in the Cartesian coordinates (left) and its parametric coordinates (right) The interpolated Cartesian coordinates and the displacement are : 


$$
\left\{\begin{array}{l}
x \\
y \\
z
\end{array}\right\}=\sum_{i=1}^{12} N_{i}\left\{\begin{array}{l}
x_{i} \\
y_{i} \\
z_{i}
\end{array}\right\} \quad \text { and } \quad\left\{\begin{array}{l}
u \\
v \\
w
\end{array}\right\}=\mathbf{N ~ q}
$$

where

$$
\begin{aligned}
& \mathbf{N}=\left[N_{1} \mathbf{I}_{3}, \ldots, N_{12} \mathbf{I}_{3}\right] \text { is the interpolation matrix } \\
& \mathbf{q}=\left[u_{1}, v_{1}, w_{1}, \ldots, u_{12}, v_{12}, w_{12}\right]^{T} \text { is the element displacement vector }
\end{aligned}
$$

In the above equation, the quantities with subscripts denote their nodal counterparts. By recalling the strain-displacement relation, namely :

$$
\mathrm{e}^{u}=\left\{\begin{array}{c}
\varepsilon_{x} \\
\varepsilon_{y} \\
\varepsilon_{z} \\
\gamma_{y z} \\
\gamma_{z x} \\
\gamma_{x y}
\end{array}\right\}=\left[\begin{array}{ccc}
\partial / \partial x & 0 & 0 \\
0 & \partial / \partial y & 0 \\
0 & 0 & \partial / \partial z \\
0 & \partial / \partial z & \partial / \partial y \\
\partial / \partial z & 0 & \partial / \partial x \\
\partial / \partial y & \partial / \partial x & 0
\end{array}\right]\left\{\begin{array}{l}
u \\
v \\
w
\end{array}\right\}
$$

and the interpolated displacement in Eqn.(2), the displacement-derived strain can be expressed as :

$$
\boldsymbol{\epsilon}=\mathbf{B} \mathbf{q}
$$

in which $\mathbf{B}$ is the strain-displacement matrix. The stiffness matrix of the standard or displacementbased element can be computed as :

$$
\mathbf{k}_{d}=\int_{v^{e}} \mathbf{B}^{T} \mathbf{C B} d v=\int_{-1}^{+1} \int_{-1}^{+1} \int_{-1}^{+1} \mathbf{B}^{T} \mathbf{C B} J d \xi d \eta d \zeta
$$

where $v^{e}$ denotes the element domain, $\mathbf{C}$ is material stiffness matrix and $J$ is Jacobian determinant. To secure the proper rank of $\mathbf{k}_{d}$, it is required to use the second, second and third order quadratures along the $\xi_{-}, \eta$ - and $\zeta$-directions, respectively. This element will be abbreviated as D12.

\section{HYBRID ELEMENT FORMULATION BASED ON ORTHOGONAL STRESS MODES}


Hellinger-Reissner functional can be derived from the potential energy functional by relaxing the stress-displacement relation (Washizu 1982). The elementwise form of the former functional is :

$$
\Pi^{e}=\int_{v^{e}}\left(-\frac{1}{2} \boldsymbol{\sigma}^{T} \mathbf{S} \boldsymbol{\sigma}+\boldsymbol{\sigma}^{T} \boldsymbol{\epsilon}\right) d v
$$

in which

$$
\begin{aligned}
& \boldsymbol{\sigma}=\left[\sigma_{x}, \sigma_{y}, \sigma_{z}, \tau_{y z}, \tau_{z x}, \tau_{x y}\right]^{T} \text { is an independently assumed stress } \\
& \mathbf{S}=\mathbf{C}^{-1} \text { is the material compliance matrix }
\end{aligned}
$$

In Eqn.(6), the load potential is omitted as it is not required for formulating the element stiffness matrix. Without losing generality, the stress can be assumed in the following form :

$$
\boldsymbol{\sigma}=\boldsymbol{\beta}_{c}+\mathbf{P}_{n} \boldsymbol{\beta}_{n}
$$

where $\mathbf{P}_{n}$ is the non-constant stress shape function matrix and $\boldsymbol{\beta}$ 's are the vectors of stress coefficients. Substituting Eqn.(4) and Eqn.(7) into Eqn.(6) results in :

$$
\Pi^{e}=-\frac{1}{2}\left\{\begin{array}{l}
\boldsymbol{\beta}_{c} \\
\boldsymbol{\beta}_{n}
\end{array}\right\}^{T}\left[\begin{array}{ll}
v_{c} \mathbf{S} & \mathbf{H}_{c n} \\
\mathbf{H}_{c n}^{T} & \mathbf{H}_{n n}
\end{array}\right]\left\{\begin{array}{l}
\boldsymbol{\beta}_{c} \\
\boldsymbol{\beta}_{n}
\end{array}\right\}+\left\{\begin{array}{l}
\boldsymbol{\beta}_{c} \\
\boldsymbol{\beta}_{n}
\end{array}\right\}^{T}\left[\begin{array}{l}
\mathbf{G}_{c} \\
\mathbf{G}_{n}
\end{array}\right] \mathbf{q}
$$

in which

$$
\begin{aligned}
& \mathbf{H}_{c n}=\int_{v^{e}} \mathbf{S} \mathbf{P}_{n} d v \text { and } \mathbf{H}_{n n}=\int_{v^{e}} \mathbf{P}_{n}^{T} \mathbf{S} \mathbf{P}_{n} d v \text { are the sub-matrices of the flexibility matrix } \\
& \mathbf{G}_{c}=\int_{v^{e}} \mathbf{B} d v \text { and } \mathbf{G}_{n}=\int_{v^{e}} \mathbf{P}_{n}^{T} \mathbf{B} d v \text { are the sub-matrices of the leverage matrix }
\end{aligned}
$$

For orthogonal constant and non-constant stress modes (Sze 1992) which have the following property :

$$
\mathbf{H}_{c n}=\mathbf{0},
$$

the above functional can be expanded as :

$$
\Pi^{e}=-\frac{v_{o}}{2} \boldsymbol{\beta}_{c}^{T} \mathbf{S} \boldsymbol{\beta}_{c}-\frac{1}{2} \boldsymbol{\beta}_{n}^{T} \mathbf{H}_{n n} \boldsymbol{\beta}_{n}+\boldsymbol{\beta}_{c}^{T} \mathbf{G}_{c} \mathbf{q}+\boldsymbol{\beta}_{n}^{T} \mathbf{G}_{n} \mathbf{q}
$$


As there is no continuity requirement on $\boldsymbol{\sigma}$ or $\boldsymbol{\beta}$ 's across the element interface, the stationary nature of Hellinger-Reissner functional with respect to $\boldsymbol{\beta}$ 's yields :

$$
\boldsymbol{\beta}_{c}=\frac{1}{v_{o}} \mathbf{C G}_{c} \mathbf{q} \quad \text { and } \quad \boldsymbol{\beta}_{n}=\mathbf{H}_{n n}^{-1} \mathbf{G}_{n} \mathbf{q}
$$

With $\boldsymbol{\beta}$ 's eliminated, the functional can be written as :

$$
\Pi^{e}=\frac{1}{2} \mathbf{q}^{T}\left(\mathbf{k}_{c}+\mathbf{k}_{n}\right) \mathbf{q}
$$

where

$$
\begin{aligned}
& \mathbf{k}_{c}=\frac{1}{v_{o}} \mathbf{G}_{c}^{T} \mathbf{C} \mathbf{G}_{c} \text { is the element stiffness matrix arising from the constant stress modes } \\
& \mathbf{k}_{n}=\mathbf{G}_{n}^{T} \mathbf{H}_{n n}^{-1} \mathbf{G}_{n} \text { is the element stiffness matrix arising from the non-constant stress modes }
\end{aligned}
$$

The advantage of adopting orthogonal stress modes is that the flexibility matrix is block-diagonal. Thus, the element computational cost can be reduced.

\section{CHOICE OF THE ASSUMED STRESS}

Undoubtedly, the most critical task of designing a hybrid stress element is choosing the nonconstant stress modes. A $2 \times 2 \times 2$ element in its parametric coordinates is first considered, see Fig.3. With the six rigid body and six constant strain modes eliminated, its displacement field can be expressed as :

$$
\begin{aligned}
& u_{\xi}=a_{1} \xi \eta+\zeta\left(a_{2} \xi+a_{3} \eta+a_{4} \xi \eta\right)+\zeta^{2}\left(a_{5}+a_{6} \xi+a_{7} \eta+a_{8} \xi \eta\right), \\
& u_{\eta}=b_{1} \xi \eta+\zeta\left(b_{2} \xi+b_{3} \eta+b_{4} \xi \eta\right)+\zeta^{2}\left(b_{5}+b_{6} \xi+b_{7} \eta+b_{8} \xi \eta\right), \\
& u_{\zeta}=c_{1} \xi \eta+\zeta\left(c_{2} \xi+c_{3} \eta+c_{4} \xi \eta\right)+\zeta^{2}\left(c_{5}+c_{6} \xi+c_{7} \eta+c_{8} \xi \eta\right)
\end{aligned}
$$

where $a_{i}$ 's, $b_{i}$ 's and $c_{i}$ 's are linear combination of the nodal displacement d.o.f.s. The strain components derived from the above displacement field are :

$$
\varepsilon_{\xi}=\frac{\partial u_{\xi}}{\partial \xi}=a_{1} \eta+\zeta\left(a_{2}+a_{4} \eta\right)+\zeta^{2}\left(a_{6}+a_{8} \eta\right), \varepsilon_{\eta}=\frac{\partial u_{\eta}}{\partial \eta}=b_{1} \xi+\zeta\left(b_{3}+b_{4} \xi\right)+\zeta^{2}\left(b_{7}+b_{8} \xi\right),
$$




$$
\begin{aligned}
& \varepsilon_{\zeta}=\frac{\partial u_{\zeta}}{\partial \zeta}=c_{2} \xi+c_{3} \eta+c_{4} \xi \eta+2 \zeta\left(c_{5}+c_{6} \xi+c_{7} \eta+c_{8} \xi \eta\right), \\
& \gamma_{\eta \zeta}=\frac{\partial u_{\eta}}{\partial \zeta}+\frac{\partial u_{\zeta}}{\partial \eta}=\left(b_{2}+c_{1}\right) \xi+b_{3} \eta+b_{4} \xi \eta+\zeta\left(2 b_{5}+c_{3}+\left(2 b_{6}+c_{4}\right) \xi+2 b_{7} \eta+2 b_{8} \xi \eta\right)+\zeta^{2}\left(c_{7}+c_{8} \xi\right), \\
& \gamma_{\zeta \xi}=\frac{\partial u_{\zeta}}{\partial \xi}+\frac{\partial u_{\xi}}{\partial \zeta}=a_{2} \xi+\left(a_{3}+c_{1}\right) \eta+a_{4} \xi \eta+\zeta\left(2 a_{5}+c_{2}+2 a_{6} \xi+\left(2 a_{7}+c_{4}\right) \eta+2 a_{8} \xi \eta\right)+\zeta^{2}\left(c_{6}+c_{8} \eta\right), \\
& \gamma_{\xi \eta}=\frac{\partial u_{\xi}}{\partial \eta}+\frac{\partial u_{\eta}}{\partial \xi}=a_{1} \xi+b_{1} \eta+\zeta\left(a_{3}+b_{2}+a_{4} \xi+b_{4} \eta\right)+\zeta^{2}\left(\left(a_{7}+b_{6}\right)+a_{8} \xi+b_{8} \eta\right)
\end{aligned}
$$

A main concern of element design is shear locking. The source of shear locking in hybrid stress elements is the assumed shear stress modes (Sze \& Ghali 1992, 1993). To alleviate the locking, the maximum number of normal stress modes that can stabilize the element are employed. Hence, all the polynomial terms in the normal strain are retained in the assumed normal stress, namely

$$
\begin{aligned}
& \sigma_{\xi}=\eta \alpha_{1}+\zeta\left(\alpha_{2}+\alpha_{3} \eta\right)+\zeta^{2}\left(\alpha_{4}+\alpha_{5} \eta\right), \sigma_{\eta}=\alpha_{6} \xi+\zeta\left(\alpha_{7}+\alpha_{8} \xi\right)+\zeta^{2}\left(\alpha_{9}+\alpha_{10} \xi\right), \\
& \sigma_{\zeta}=\alpha_{11} \xi+\alpha_{12} \eta+\alpha_{13} \xi \eta+\zeta\left(\alpha_{14}+\alpha_{15} \xi+\alpha_{16} \eta+\alpha_{17} \xi \eta\right)
\end{aligned}
$$

in which $\alpha$ 's are the stress coefficients. With these seventeen stress modes, the unstabilized strain modes (Pian \& Chen 1983) are :

$$
\gamma_{\eta \zeta}=\left(b_{2}+c_{1}\right) \xi+\zeta\left(2 b_{5}+2 b_{6} \xi\right), \gamma_{\zeta \xi}=\left(a_{3}+c_{1}\right) \eta+\zeta\left(2 a_{5}+2 a_{7} \eta\right), \gamma_{\xi \eta}=\zeta\left(a_{3}+b_{2}\right)+\zeta^{2}\left(a_{7}+b_{6}\right)
$$

which can be suppressed by including the following shear stress modes :

$$
\tau_{\eta \zeta}=\alpha_{18} \xi+\alpha_{19} \zeta+\alpha_{20} \zeta \xi, \tau_{\zeta \xi}=\alpha_{21} \eta+\alpha_{22} \zeta+\alpha_{23} \zeta \eta, \tau_{\xi \eta}=\alpha_{24} \zeta
$$

As the stress components given in Eqn.(15) and Eqn.(17) are symmetric with respect to $\xi$ and $\eta$, the resulting element will be insensitive to different connectivity sequences (Sze, Chow \& Chen 1992). Moreover, the twenty-four non-constant stress modes together with the six constant stress modes constitute a minimal stress field that can secure the element rank. The latter equals the difference between the number of the nodal displacement d.o.f.s and the number of rigid body modes (Pian \& Chen 1983). 
To generalize the above non-constant stress modes to other element configurations, they are transformed from the parametric to the Cartesian coordinates using the covariant base vectors evaluated at the element original (Pian \& Sumihara 1984; Pian 1985). This transformation is also an effective way to secure the frame-invariance of the element (Sze, Chow \& Chen 1992). The transformation can be derived as (Fung 1965) :

$$
\boldsymbol{\sigma}=\mathbf{T} \widetilde{\boldsymbol{\sigma}}
$$

where

$$
\begin{aligned}
& \tilde{\boldsymbol{\sigma}}=\left[\sigma_{\xi}, \sigma_{\eta}, \sigma_{\zeta}, \tau_{\eta \zeta}, \tau_{\zeta \xi}, \tau_{\xi \eta}\right]^{T} \\
& \mathbf{T}=\left[\begin{array}{cccccc}
J_{11}^{2} & J_{21}^{2} & J_{31}^{2} & 2 J_{21} J_{31} & 2 J_{31} J_{11} & 2 J_{11} J_{21} \\
J_{12}^{2} & J_{22}^{2} & J_{32}^{2} & 2 J_{22} J_{32} & 2 J_{32} J_{12} & 2 J_{12} J_{22} \\
J_{13}^{2} & J_{23}^{2} & J_{33}^{2} & 2 J_{23} J_{33} & 2 J_{33} J_{13} & 2 J_{13} J_{23} \\
J_{12} J_{13} & J_{22} J_{23} & J_{32} J_{33} & J_{22} J_{33}+J_{32} J_{23} & J_{32} J_{13}+J_{12} J_{33} & J_{12} J_{23}+J_{22} J_{13} \\
J_{13} J_{11} & J_{23} J_{21} & J_{33} J_{31} & J_{23} J_{31}+J_{31} J_{23} & J_{33} J_{11}+J_{11} J_{33} & J_{13} J_{21}+J_{21} J_{13} \\
J_{11} J_{12} & J_{21} J_{22} & J_{31} J_{32} & J_{21} J_{32}+J_{31} J_{22} & J_{31} J_{12}+J_{11} J_{32} & J_{11} J_{22}+J_{21} J_{12}
\end{array}\right] \\
& {\left[J_{i j}\right]=\left[\left.\begin{array}{lll}
\partial x / \partial \xi & \partial y / \partial \xi & \partial z / \partial \xi \\
\partial x / \partial \eta & \partial y / \partial \eta & \partial z / \partial \eta \\
\partial x / \partial \zeta & \partial y / \partial \zeta & \partial z / \partial \zeta
\end{array}\right|_{\xi=\eta=\zeta=0}\right.}
\end{aligned}
$$

The complete non-constant stress field can be expressed as :

$$
\begin{aligned}
\boldsymbol{\sigma}=\zeta \mathbf{I}_{6} \boldsymbol{\beta}_{\zeta}+\xi \mathbf{P}_{\xi} \boldsymbol{\beta}_{\xi}+\eta \mathbf{P}_{\eta} \boldsymbol{\beta}_{\eta}+\zeta \xi \mathbf{P}_{\xi} \boldsymbol{\beta}_{\zeta \xi}+\zeta \eta \mathbf{P}_{\eta} \boldsymbol{\beta}_{\zeta \eta} \\
+\zeta^{2} \mathbf{P}_{\zeta} \boldsymbol{\beta}_{\zeta \zeta}+\xi \eta \mathbf{T}_{3} \boldsymbol{\beta}_{\xi \eta}+\zeta \xi \eta \mathbf{T}_{3} \boldsymbol{\beta}_{\zeta \xi \eta}+\zeta^{2} \xi \mathbf{T}_{2} \boldsymbol{\beta}_{\zeta \zeta \xi}+\zeta^{2} \eta \mathbf{T}_{1} \boldsymbol{\beta}_{\zeta \zeta \eta}
\end{aligned}
$$

in which

$$
\mathbf{P}_{\xi}=\left[\mathbf{T}_{2}, \mathbf{T}_{3}, \mathbf{T}_{4}\right], \mathbf{P}_{\eta}=\left[\mathbf{T}_{1}, \mathbf{T}_{3}, \mathbf{T}_{4}\right], \mathbf{P}_{\zeta}=\left[\mathbf{T}_{1}, \mathbf{T}_{2}\right] \text { and } \mathbf{T}_{i} \text { is the i-th column of } \mathbf{T}
$$

As the $\zeta$-modes associated with $\boldsymbol{\beta}_{\zeta}$ present in all stress components, the stress basis remains unchanged whether the $\zeta$-modes are transformed or not (Sze, Chow \& Chen 1992). Comparing Eqn.(7) with Eqn.(19) and orthogonalizing the stress modes according to the requirement laid down in Eqn.(9), we have 


$$
\begin{gathered}
\mathbf{P}_{n}=\frac{1}{J}\left[\zeta \mathbf{I}_{6}, \xi \mathbf{P}_{\xi}, \eta \mathbf{P}_{\eta}, \zeta \xi \mathbf{P}_{\xi}, \zeta \eta \mathbf{P}_{\eta},\left(\zeta^{2}-\frac{1}{3}\right) \mathbf{P}_{\zeta}, \xi \eta \mathbf{T}_{3}, \zeta \xi \eta \mathbf{T}_{3},\left(\zeta^{2}-\frac{1}{3}\right) \xi \mathbf{T}_{2},\left(\zeta^{2}-\frac{1}{3}\right) \eta \mathbf{T}_{1}\right], \\
\boldsymbol{\beta}_{n}=\left[\boldsymbol{\beta}_{\zeta}^{T}, \boldsymbol{\beta}_{\xi}^{T}, \boldsymbol{\beta}_{\eta}^{T}, \boldsymbol{\beta}_{\zeta \xi}^{T}, \boldsymbol{\beta}_{\zeta \eta}^{T}, \boldsymbol{\beta}_{\zeta \zeta}^{T}, \beta_{\xi \eta}, \beta_{\zeta \xi \eta}, \beta_{\zeta \zeta \xi}, \beta_{\zeta \zeta \eta}\right]^{T}
\end{gathered}
$$

With $\mathbf{P}_{n}$ finalized, the element stiffness can be computed, see Eqn.(12). The resulting element will be abbreviated as $\mathrm{H} 12$.

\section{AN EFFICIENT ALTERNATIVE USING ADMISSIBLE MATRIX FORMULATION}

To form H12, the $24 \times 24 \mathbf{H}_{n n}$ matrix has to be inverted. It has been shown in the admissible formulation that the matrix entries in $\mathbf{H}_{n n}$ can be varied without jeopardizing the patch test fulfillment provided that $\mathbf{H}_{n n}$ is still positive-definite (Sze 1992, 1996). As the highest element accuracy is often yielded by using regular meshes, only the $\mathbf{H}_{n n}$-terms which do not vanish when the element assumes the geometry of a rectangular prism are retained. In this light, $\mathbf{H}_{n n}$ becomes :

$$
\mathbf{H}_{n n}=\operatorname{diag} .\left\{v_{\zeta} \mathbf{S}, v_{\xi} \mathbf{H}_{\xi}, v_{\eta} \mathbf{H}_{\eta}, v_{\zeta \xi} \mathbf{H}_{\xi}, v_{\zeta \eta} \mathbf{H}_{\eta}, v_{\zeta \zeta} \mathbf{H}_{\zeta}, v_{\xi \eta} H_{3}, v_{\zeta \xi \eta} H_{3}, v_{\zeta \zeta \xi} H_{2}, v_{\zeta \zeta \eta} H_{1}\right\}
$$

where

$$
\begin{aligned}
& \mathbf{H}_{\xi}=\mathbf{P}_{\xi}^{T} \mathbf{S P}_{\xi}, \mathbf{H}_{\eta}=\mathbf{P}_{\eta}^{T} \mathbf{S P}_{\eta}, \mathbf{H}_{\zeta}=\mathbf{P}_{\zeta}^{T} \mathbf{S P}_{\zeta}, H_{i}=\mathbf{T}_{i}^{T} \mathbf{S} \mathbf{T}_{i} \\
& \left\{v_{\zeta}, v_{\xi}, v_{\eta}, v_{\zeta \xi}, v_{\zeta \eta}\right\}=\int_{v^{e}} \frac{1}{J^{2}}\left\{\zeta^{2}, \xi^{2}, \eta^{2}, \zeta^{2} \xi^{2}, \zeta^{2} \eta^{2}\right\} d v \\
& \left\{v_{\zeta \zeta}, v_{\xi \eta}, v_{\zeta \xi \eta}, v_{\zeta \zeta \xi}, v_{\zeta \zeta \eta}\right\}=\int_{v^{e}} \frac{1}{J^{2}}\left\{\left(\zeta^{2}-\frac{1}{3}\right)^{2}, \xi^{2} \eta^{2}, \zeta^{2} \xi^{2} \eta^{2},\left(\zeta^{2}-\frac{1}{3}\right)^{2} \xi^{2},\left(\zeta^{2}-\frac{1}{3}\right)^{2} \eta^{2}\right\} d v
\end{aligned}
$$

It can be seen that the formation of $\mathbf{H}_{\xi}, \mathbf{H}_{\eta}, \mathbf{H}_{\zeta}$ and $H_{i}$ 's does not involve integration. With the above block diagonal $\mathbf{H}_{n n}$, the element stiffness $\mathbf{k}_{c}+\mathbf{k}_{n}$ given in Eqn.(12) can be expanded :

$$
\begin{aligned}
\mathbf{k}= & \frac{1}{v_{o}} \mathbf{G}_{c}^{T} \mathbf{C G}_{c}+\frac{1}{v_{\zeta}} \mathbf{G}_{\zeta}^{T} \mathbf{C G}_{\zeta}+\frac{1}{v_{\xi}} \mathbf{G}_{\xi}^{T} \mathbf{H}_{\xi}^{-1} \mathbf{G}_{\xi}+\frac{1}{v_{\eta}} \mathbf{G}_{\eta}^{T} \mathbf{H}_{\eta}^{-1} \mathbf{G}_{\eta}+\frac{1}{v_{\zeta \zeta}} \mathbf{G}_{\zeta \zeta}^{T} \mathbf{H}_{\zeta}^{-1} \mathbf{G}_{\zeta \xi}+\frac{1}{v_{\zeta \eta}} \mathbf{G}_{\zeta \eta}^{T} \mathbf{H}_{\eta}^{-1} \mathbf{G}_{\zeta \eta} \\
& +\frac{1}{v_{\zeta \zeta}} \mathbf{G}_{\zeta \zeta}^{T} \mathbf{H}_{\zeta}^{-1} \mathbf{G}_{\zeta \zeta}+\frac{1}{v_{\xi \eta} H_{3}} \mathbf{G}_{\xi \eta}^{T} \mathbf{G}_{\xi \eta}+\frac{1}{v_{\zeta \xi \eta} H_{3}} \mathbf{G}_{\zeta \zeta \eta}^{T} \mathbf{G}_{\zeta \xi \eta}+\frac{1}{v_{\zeta \zeta \xi} H_{2}} \mathbf{G}_{\zeta \zeta \zeta}^{T} \mathbf{G}_{\zeta \zeta \xi}+\frac{1}{v_{\zeta \zeta \eta} H_{1}} \mathbf{G}_{\zeta \zeta \eta}^{T} \mathbf{G}_{\zeta \zeta \eta}
\end{aligned}
$$

where

$$
\mathbf{G}_{\zeta}=\int_{v^{e}} \frac{\zeta}{J} \mathbf{B} d v, \mathbf{G}_{\xi}=\mathbf{P}_{\xi}^{T} \int_{v^{e}} \frac{\xi}{J} \mathbf{B} d v, \mathbf{G}_{\eta}=\mathbf{P}_{\eta}^{T} \int_{v^{e}} \frac{\eta}{J} \mathbf{B} d v, \mathbf{G}_{\zeta \xi}=\mathbf{P}_{\zeta \xi}^{T} \int_{v^{e}} \frac{\zeta \xi}{J} \mathbf{B} d v,
$$




$$
\begin{aligned}
& \mathbf{G}_{\zeta \eta}=\mathbf{P}_{\eta}^{T} \int_{v^{e}} \frac{\zeta \eta}{J} \mathbf{B} d v, \mathbf{G}_{\zeta \zeta}=\mathbf{P}_{\zeta}^{T} \int_{v^{e}} \frac{1}{J}\left(\zeta^{2}-\frac{1}{3}\right) \mathbf{B} d v, \mathbf{G}_{\xi \eta}=\mathbf{T}_{3}^{T} \int_{v^{e}} \frac{\xi \eta}{J} \mathbf{B} d v, \\
& \mathbf{G}_{\zeta \xi \eta}=\mathbf{T}_{3}^{T} \int_{v^{e}} \frac{\zeta \xi \eta}{J} \mathbf{B} d v, \mathbf{G}_{\zeta \zeta \xi}=\mathbf{T}_{2}^{T} \int_{v^{e}} \frac{\xi}{J}\left(\zeta^{2}-\frac{1}{3}\right) \mathbf{B} d v, \mathbf{G}_{\zeta \zeta \eta}=\mathbf{T}_{1}^{T} \int_{v^{e}} \frac{\eta}{J}\left(\zeta^{2}-\frac{1}{3}\right) \mathbf{B} d v
\end{aligned}
$$

This element will be abbreviated as H12a. Instead of inverting the $24 \times 24 \mathbf{H}_{n n}$-matrix in H12, only two $3 \times 3\left(\mathbf{H}_{\xi}\right.$ and $\left.\mathbf{H}_{\eta}\right)$ and one $2 \times 2\left(\mathbf{H}_{\zeta}\right)$ sub-matrices have to be inverted in H21a. Vectors of stress coefficients are retrieved from the element displacement vector via Eqn.(11) which can be expressed as :

$$
\begin{aligned}
& \boldsymbol{\beta}_{c}=\frac{1}{v_{c}} \mathbf{C G}_{c} \mathbf{q}, \boldsymbol{\beta}_{\zeta}=\frac{1}{v_{\zeta}} \mathbf{C G}_{\zeta} \mathbf{q}, \boldsymbol{\beta}_{\xi}=\frac{1}{v_{\xi}} \mathbf{H}_{\xi}^{-1} \mathbf{G}_{\xi} \mathbf{q}, \boldsymbol{\beta}_{\eta}=\frac{1}{v_{\eta}} \mathbf{H}_{\eta}^{-1} \mathbf{G}_{\eta} \mathbf{q}, \boldsymbol{\beta}_{\zeta \xi}=\frac{1}{v_{\zeta \xi}} \mathbf{H}_{\xi}^{-1} \mathbf{G}_{\zeta \xi} \mathbf{q}, \\
& \boldsymbol{\beta}_{\zeta \eta}=\frac{1}{v_{\zeta \eta}} \mathbf{H}_{\zeta \eta}^{-1} \mathbf{G}_{\zeta \eta} \mathbf{q}, \boldsymbol{\beta}_{\zeta \zeta}=\frac{1}{v_{\zeta \zeta}} \mathbf{H}_{\zeta}^{-1} \mathbf{G}_{\zeta \zeta} \mathbf{q}, \beta_{\xi \eta}=\frac{1}{v_{\xi \eta} H_{3}} \mathbf{G}_{\xi \eta} \mathbf{q}, \beta_{\zeta \xi \eta}=\frac{1}{v_{\zeta \xi \eta} H_{3}} \mathbf{G}_{\zeta \xi \eta} \mathbf{q}, \\
& \beta_{\zeta \xi \xi}=\frac{1}{v_{\zeta \zeta \xi} H_{2}} \mathbf{G}_{\zeta \zeta \xi} \mathbf{q}, \boldsymbol{\beta}_{\zeta \zeta \eta}=\frac{1}{v_{\zeta \zeta \eta} H_{1}} \mathbf{G}_{\zeta \zeta \eta} \mathbf{q}
\end{aligned}
$$

\section{NUMERICAL TESTS}

As the elements are designed primarily for beam and column analysis, a number of popular beam problems will be analysed by D12, H12 and H12a which have been elaborated in Sections 2, 4 and 5 , respectively.

Single-Element Cantilever - A 10 unit-long cantilever is loaded by end bending and end shear force as shown in Fig.4. The analytical solutions for this problem are extracted from (Timoshenko $\&$ Goodier 1982). Two cross sections of dimension $2 \times 2$ and $0.2 \times 0.2$ are considered. The cantilever is modelled by one element. As the element is a rectangular prism, H12 and H12a are identical. The predicted tip deflections are listed in Table 1. Though the element displacement is only quadratic in $x, \mathrm{H} 12$ and H12a yield excellent deflections even for the end shear-loaded beam whose deflection is a cubic function of $x$. It can also be seen that $\mathrm{H} 12$ and $\mathrm{H} 12 \mathrm{a}$ are not susceptible to the element aspect ratio. The predicted stresses for the $2 \times 2 \times 10$ cantilever under pure bending and end shear at the $2 \times 2 \times 3$ integration points closest to $x$-axis are given in Table 2 and Table 3 . Owing to the limitation of the element interpolation, the parabolic transverse shear stress can only be approximated by the one which is constant along the transverse direction. In this light, all the predicted stresses of $\mathrm{H} 12 / \mathrm{H} 12 \mathrm{a}$ are exact whereas D12 gives erroneous predictions. 

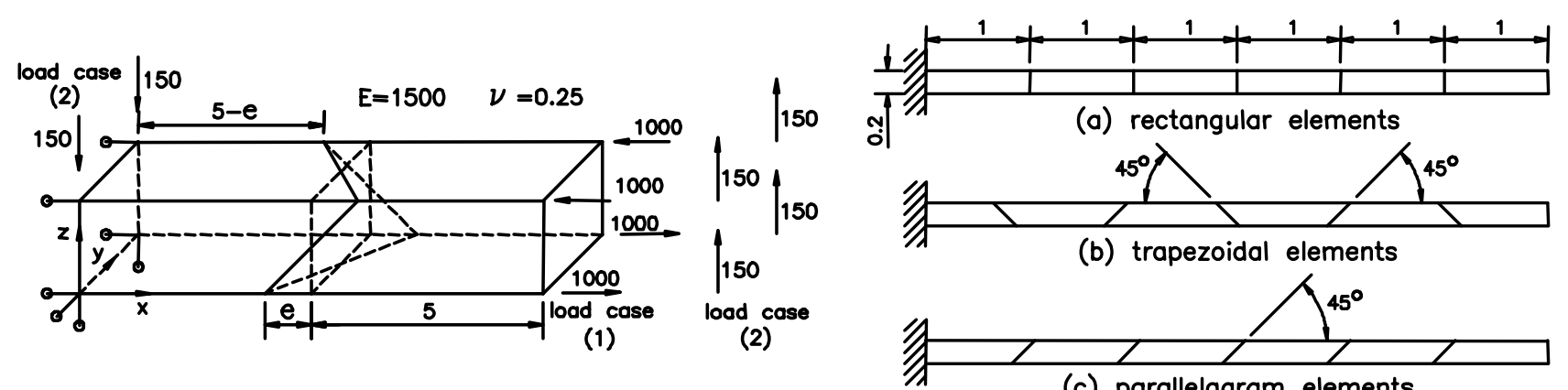

(c) parallelagram elements

Fig.4. A $2 \times 2 \times 10$ cantilever subjected to end bending and end shear force Slender (left); slender cantilever modelled by three different meshes (right)

Table 1. Predicted tip deflections for the single-element cantilever problem, see Fig.4 (left)

\begin{tabular}{cccccc}
\hline & \multicolumn{2}{c}{ cantilever dimension $: 2 \times 2 \times 10$} & & \multicolumn{2}{c}{ cantilever dimension : $0.2 \times 0.2 \times 10$} \\
\cline { 2 - 3 } & bending & shear & & bending & shear \\
\hline D12 & 90.91 & 78.74 & & 90,909 & 683,311 \\
H12/H12a & 100 & 102.50 & & 100,000 & $1,000,250$ \\
analytical & 100 & $103.75^{*}$ & & 100,000 & $1,000,375^{*}$ \\
\hline
\end{tabular}

* if the transverse shear is assumed to be constant instead of parabolic, the deflections should be 102.50 and $1,000,250$.

Table 2. Predicted stresses for the $2 \times 2 \times 10$ single-element cantilever under pure bending at the $2 \times 2 \times 3$ integration points closest to $\mathrm{x}$-axis for the cantilever problem, see Fig.4 (left)

\begin{tabular}{cccccccc}
\hline & & $\sigma_{x}$ & $\sigma_{y}$ & $\sigma_{z}$ & $\tau_{y z}$ & $\tau_{z x}$ & $\tau_{x y}$ \\
\hline \multirow{4}{*}{$\mathrm{x} / 5=1-\sqrt{ } .6$} & D12 & 1732.1 & 157.46 & 472.38 & -157.46 & 0.0000 & 0.0000 \\
& H12/H12a & 1732.1 & 0.0000 & 0.0000 & 0.0000 & 0.0000 & 0.0000 \\
& analytical & 1732.1 & 0 & 0 & 0 & 0 & 0 \\
\hline \multirow{3}{*}{$\mathrm{x} / 5=1$} & D12 & 1732.1 & 157.46 & 472.38 & -157.46 & 0.0000 & 0.0000 \\
& H12/H12a & 1732.1 & 0.0000 & 0.0000 & 0.0000 & 0.0000 & 0.0000 \\
& analytical & 1732.1 & 0 & 0 & 0 & 0 & 0 \\
\hline \multirow{3}{*}{$\mathrm{x} / 5=1+\sqrt{ } .6$} & D12 & 1732.1 & 157.46 & 472.38 & -157.46 & 0.0000 & 0.0000 \\
& H12/H12a & 1732.1 & 0.0000 & 0.0000 & 0.0000 & 0.0000 & 0.0000 \\
& analytical & 1732.1 & 0 & 0 & 0 & 0 & 0 \\
\hline
\end{tabular}


Table 3. Predicted stresses for the $2 \times 2 \times 10$ single-element cantilever under end shear at the $2 \times 2 \times 3$ integration points closest to $\mathrm{x}$-axis for the cantilever problem, see Fig.4 (left)

\begin{tabular}{cccccccc}
\hline & & $\sigma_{x}$ & $\sigma_{y}$ & $\sigma_{z}$ & $\tau_{y z}$ & $\tau_{z x}$ & $\tau_{x y}$ \\
\hline \multirow{4}{*}{$\mathrm{x} / 5=1-\sqrt{ } .6$} & $\mathrm{D} 12$ & 1656.3 & 151.51 & 451.95 & -150.22 & 343.49 & -4.7893 \\
& H12/H12a & 2305.3 & 0.0000 & 0.0000 & 0.0000 & -150.00 & 0.0000 \\
& analytical & 2305.3 & 0 & 0 & 0 & $-150^{*}$ & 0 \\
\hline \multirow{3}{*}{$\mathrm{x} / 5=1$} & $\mathrm{D} 12$ & 1299.0 & 118.11 & 354.28 & -118.09 & -91.858 & -4.7893 \\
& H12/H12a & 1299.0 & 0.0000 & 0.0000 & 0.0000 & -150.00 & 0.0000 \\
& analytical & 1299.0 & 0 & 0 & 0 & $-150^{*}$ & 0 \\
\hline \multirow{3}{*}{$\mathrm{x} / 5=1+\sqrt{ } .6$} & D12 & 941.8 & 84.681 & 256.62 & -85.967 & 343.49 & -4.7893 \\
& H12/H12a & 292.8 & 0.0000 & 0.0000 & 0.0000 & -150.00 & 0.0000 \\
& analytical & 292.8 & 0 & 0 & 0 & $-150^{*}$ & 0 \\
\hline
\end{tabular}

* transverse shear is assumed to be constant instead of parabolic, $\tau_{z x}$

Two-Element Cantilever - The $2 \times 2 \times 10$ cantilever is now modelled by two elements. This problem aims at testing the effect of element distortion on the element accuracy. The elements are distorted by shifting the nodes at the mid-span by amount "e" as noted in Fig.4 (left). The end deflection and the bending stress at the $\zeta=0$ integration point closest to the $\mathrm{x}$-axis of the element at the left hand side are shown in Fig.5. Both $\mathrm{H} 12$ and H12a obtain accurate results whereas D12 deteriorates quickly with increasing "e".
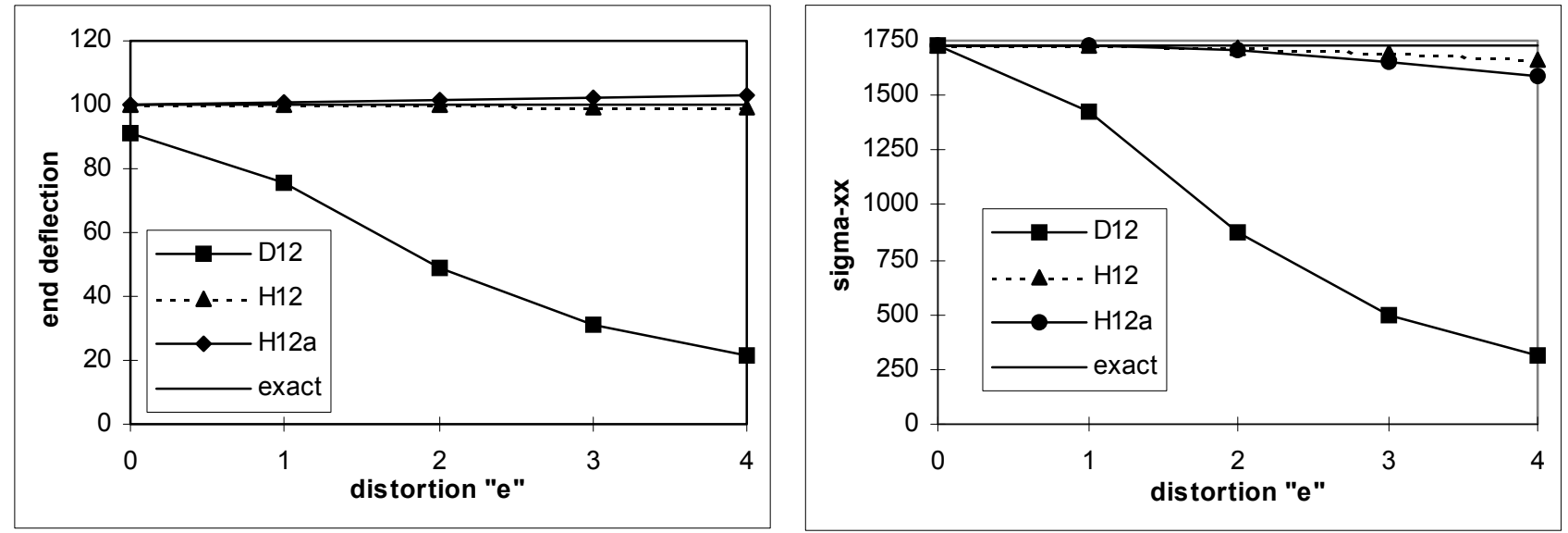

Fig.5. Predicted end deflection (left) and bending stress $\sigma_{x x}$ (right) for the $2 \times 2 \times 10$ cantilever subjected to end bending, see Fig.3 (left)

Slender Cantilever This problem is defined in Fig.4 (right). The cantilever is subjected to unit inplane and out-of-plane end shear loads. The material properties are : $E=10^{7}$ and $v=0.3$. The predicted tip deflections along the loading directions are listed in Table 4. While substantial errors are observed in D12 with the mesh distorted, H12 and H12a remain accurate. The reference solution is extracted from (MacNeal \& Harder 1985). 
Table 4. Predicted tip deflections for the slender cantilever problem, see Fig.4 (right)

\begin{tabular}{|c|c|c|c|c|c|c|}
\hline & \multicolumn{3}{|c|}{ in-plane loading } & \multicolumn{3}{|c|}{ out-of-plane loading } \\
\hline & rectangular & $\begin{array}{c}\text { parallelogra } \\
\mathrm{m}\end{array}$ & trapezoidal & rectangular & parallelogram & trapezoidal \\
\hline D12 & 0.0955 & 0.0372 & 0.0538 & 0.3473 & 0.3640 & 0.3646 \\
\hline $\mathrm{H} 12$ & 0.1075 & 0.1075 & 0.1074 & 0.4298 & 0.4304 & 0.4303 \\
\hline $\mathrm{H} 12 \mathrm{a}$ & 0.1075 & 0.1075 & 0.1075 & 0.4298 & 0.4303 & 0.4305 \\
\hline $\begin{array}{c}\text { referenc } \\
\mathrm{e}\end{array}$ & & 0.1081 & & & 0.4321 & \\
\hline
\end{tabular}

Twisted Beam Problem Fig.6 (left) shows a $90^{\circ}$ pre-twisted beam modelled by a single layer of elements. The meshes considered consist of $1 \times 3,1 \times 6,2 \times 6$ elements. The beam tip deflections in the directions of loading are given in Table 5. Again, H12 and H12a yield higher accuracy than D12. The reference solution is extracted from (MacNeal \& Harder 1985).

Table 5. Predicted tip deflections for the pre-twisted cantilever problem, see Fig.6 (left)

\begin{tabular}{ccccccc}
\hline & & in-plane loading & & \multicolumn{3}{c}{ out-of-plane loading } \\
\cline { 3 - 6 } & $\begin{array}{c}1 \times 3 \\
\text { elements }\end{array}$ & $\begin{array}{c}2 \times 3 \\
\text { elements }\end{array}$ & $\begin{array}{c}2 \times 6 \\
\text { elements }\end{array}$ & $\begin{array}{c}1 \times 3 \\
\text { elements }\end{array}$ & $\begin{array}{c}2 \times 3 \\
\text { elements }\end{array}$ & $\begin{array}{c}2 \times 6 \\
\text { elements }\end{array}$ \\
\hline D12 & 0.004221 & 0.004298 & 0.004877 & 0.001427 & 0.001463 & 0.001603 \\
H12 & 0.005438 & 0.005409 & 0.005435 & 0.001752 & 0.001743 & 0.001753 \\
H12a & 0.005438 & 0.005409 & 0.005436 & 0.001752 & 0.001743 & 0.001753 \\
\hline reference & & 0.005424 & & & 0.001754 & \\
\hline
\end{tabular}

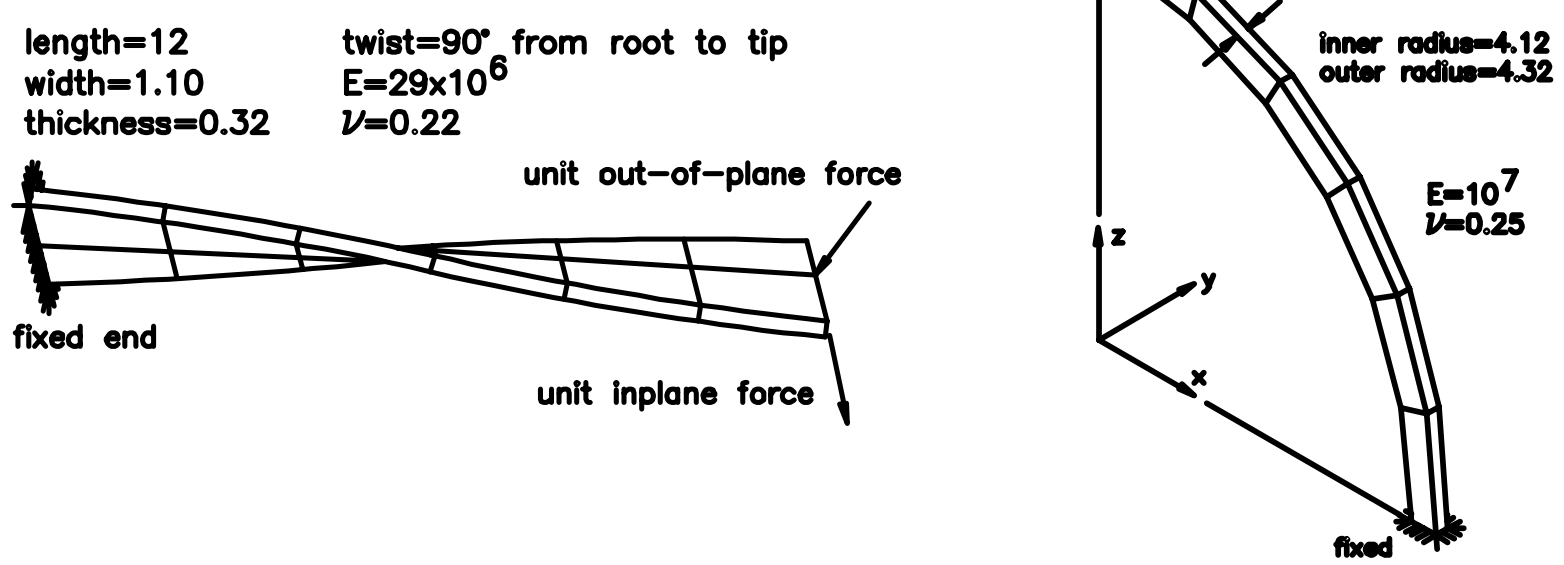

Fig.6. Pre-twisted beam modelled by $2 \times 6$ elements (left) and curved Beam modelled by $1 \times 1 \times 6$ elements (right)

Curved Beam This problem is depicted in Fig.6 (right). The beam is modelled by five different meshes. In the densest mesh, 2160 elements are employed. The reason of adopting so many elements is that our finite element predictions do not in very good agreement with the reference (not necessarily exact) solutions 0.08734 and 0.5022 given by (MacNeal \& Harder 1985). Nevertheless, 
the differences of $\mathrm{H} 12$ and $\mathrm{H} 12 \mathrm{a}$ from the reference solutions are around 5\% even with the coarsest $1 \times 1 \times 3$ mesh, see Table 6 .

Table 6. Predicted tip deflections for curved beam problem, see Fig.6 (right)

\begin{tabular}{ccccccc}
\hline \multicolumn{2}{c}{ number of elements } & $1 \times 1 \times 3$ & $1 \times 1 \times 6$ & $1 \times 1 \times 9$ & $3 \times 3 \times 18$ & $3 \times 4 \times 180$ \\
\hline \multirow{2}{*}{ in-plane loading } & $\mathrm{D} 12$ & 0.02647 & 0.07206 & 0.07981 & 0.08740 & 0.08834 \\
& $\mathrm{H} 12$ & 0.09081 & 0.08933 & 0.08893 & 0.08840 & 0.08850 \\
& $\mathrm{H} 12 \mathrm{a}$ & 0.09078 & 0.08933 & 0.08893 & 0.08840 & 0.08850 \\
\hline \multirow{2}{*}{ out-of-plane loading } & $\mathrm{D} 12$ & 0.4169 & 0.4365 & 0.4308 & 0.4756 & 0.4889 \\
& $\mathrm{H} 12$ & 0.4749 & 0.4771 & 0.4775 & 0.4829 & 0.4915 \\
& $\mathrm{H} 12 \mathrm{a}$ & 0.4796 & 0.4773 & 0.4774 & 0.4829 & 0.4915 \\
\hline
\end{tabular}

\section{CLOSURE}

A hybrid stress twelve-node for beam/column analysis is presented. The assumed stress field with the minimal modes is derived by observing rank sufficiency, frame invariance and insensitivity to connectivity sequence of the element. To reduce computational cost, the admissible matrix formulation is incorporated that enforces high sparsity in the flexibility matrix. To form the element, only two $3 \times 3$ and one $2 \times 2$ symmetric matrices have to be inverted. A number of popular beam bending problems are examined. It is found that the proposed elements yield much more accurate displacement and stress predictions than the standard displacement element. Moreover, the induced sparsity in the flexibility matrix does not have any pronounced adverse effect on the element accuracy.

The present element can be extended to geometric and material nonlinear analyses by employing the frameworks in (Atluri 1973; Pian 1976; Sze \& Zheng 1999) for hybrid stress models. On the other hand, dynamic analyses can be tackled simply by including the mass and damping matrices which depend only on the displacement interpolation and independent of the stress assumption.

Acknowledgment - The financial support of the Research Grant Council of Hong Kong in the form of an Earmarked Research Grant is gratefully acknowledged. 


\section{REFERENCES}

Atluri, S.N. 1973 : “On the hybrid stress finite element model for incremental analysis of large deflection problems", Inter.J.Solids Struct., 9, 1177-1191

Ausserer, M.F., Lee, S.W. 1988 : “An eighteen-node solid element for thin shell analysis", Inter.J. Numer. Methods Engrg., 26, 1345-1364

Belytschko, T., Tsay, C.S., Liu, W.K. 1981 : “A stabilization matrix for the bilinear Mindlin plate element”, Computer Methods Appl.Mech.Engrg., 29, 313-327

Belytschko, T., Ong, S.J., Liu, W.K., Kennedy, J.M. 1984 : "Hourglass control in linear and nonlinear problems", Computer Methods Appl.Mech.Engrg., 43, 251-276

Belytschko, T., Wong, B.K., Stolarski H. 1989 : “Assumed strain stabilization procedure for the 9node Lagrange shell element”, Inter.J.Numer.Methods.Engrg., 28, 385-414

Bathe, K.J., Dvorkin, E.N. 1985 : “A four-node plate bending element based on Mindlin/Reissner plate theory and a mixed interpolation”, Inter.J.Numer.Methods Engrg., 21, 367-383

Fung, Y.C. 1965. Foundation of Solid Mechanics, Prentice-Hall, New Jersey

Huang, H.C., Hinton, E. 1986 : "A new nine node degenerated shell element with enhanced membrane and shear interpolation", Inter.J.Numer.Methods Engrg., 22, 73-92

Kavanagh, K.T., Key, S.W. 1972 : “A note on selective and reduced integration techniques in finite element method", Inter.J.Numer.Methods Engrg., 4, 148-150

Kim, Y.H., Lee, S.W. 1988 : “A solid element formulation for large deflection analysis of composite shell structures", Computers \& Structures, 30, 269-274

MacNeal, R.H., Harder, R.L. 1985. "A proposed standard set of problems to test finite element accuracy", Finite Elements in Analysis \& Design, 1, 3-20

Malkus, D.S., Hughes, T.J.R. 1978 : "Mixed finite element methods - reduced and selective integration techniques : a unification of concepts", Computer Methods Appl.Mech.Engrg., 15, 63-81

Park, H.C., Cho, C., Lee, S.W. 1995 : “An efficient assumed strain element model with six dof per node for geometrically nonlinear shells", Inter.J.Numer.Methods Engrg., 38, 4101-4122

Pian, T.H.H. 1964 : "Derivation of element stiffness matrices by assumed stress distributions", AIAA J., 2, 1333-1336

Pian, T.H.H. 1976 : "Variational principles for incremental finite element methods", J.Franklin Institute, 302, 473-488

Pian, T.H.H., Sumihara, K. 1984 : "Rational approach for assumed stress finite elements", Inter.J.Numer. Methods Engrg., 20, 1685-1695 
Pian, T.H.H. 1985 : "Finite elements based on consistently assumed stresses and displacements", Finite Elements in Analysis \& Design, 1, 131-140

Pian, T.H.H., Chen, D.-P. 1983 : "On the suppression of zero energy deformation modes", Inter.J.Numer. Methods Engrg., 19, 1741-1752

Simo, J.C., Rifai, M.S. 1990. "A class of mixed assumed strain methods and the method of incompatible modes", Inter.J.Numer.Methods Engrg., 29, 1595-1638

Sze, K.Y. 1992 : "Efficient formulation of robust hybrid elements using orthogonal stress/strain interpolants and admissible matrix formulation”, Inter.J.Numerical Methods Engrg., 35, 1-20

Sze, K.Y., Ghali, A. 1992 : "A two-field solid element suiting thin-mesh analysis by admissible matrix formulation”, Engineering Computations, 9, 649-668

Sze, K.Y., Chow, C.L., Chen, W.-J. 1992. "On invariance of isoparametric hybrid elements", Commun.Appl. Numer.Methods, 8, 385-406

Sze, K.Y., Ghali, A. 1993 : "An hexahedral element for plates, shells and beams by selective scaling”, Inter.J.Numer. Methods Engrg., 36, 1519-1540

Sze, K.Y. 1996 : “Admissible matrix formulation : from orthogonal approach to explicit hybridstabilization", Finite Elements in Analysis \& Design, 24, 1-30

Sze, K.Y., Yi, S., Tay, M.H. 1997 : “An explicit hybrid-stabilized eighteen-node solid element for thin shell analysis”, Inter.J.Numerical Methods Engrg., 40, 1839-1856

Sze, K.Y., Zheng, S.-J. 1999 : “A hybrid stress nine-node degenerated shell element for geometric nonlinear analysis", to appear in Computational Mechanics, 23

Taylor, R.L., Beresford, P.J., Wilson, E.L. 1976 : "A non-conforming element for stress analysis", Inter.J. Numer.Methods Engrg., 10, 1211-1219

Timoshenko S.P., Goodier, J.N. 1982. Theory of Elasticity, 3rd Edn., McGraw-Hill, New York

Washizu, K. 1982 : Variational Method in Elasticity and Plasticity, 3rd Edn, Pergammon Press, Oxford 